\title{
Superpartners and Future Colliders
}

\author{
G.L.Kane \\ Randall Physics Laboratory \\ University of Michigan \\ Ann Arbor, MI 48109
}

\begin{abstract}
This talk focuses on two aspects of the search for supersymmetry. First I argue that if the breaking of the electroweak symmetry is indeed explained by supersymmetry, then it is very likely that superpartners will be produced at Fermilab. Second, once superpartners are found we will move to the next stage where we need to learn the implications of how supersymmetry is realized for the underlying theory, e.g. string theory. The connection between data and the underlying theory is via the supersymmetry soft-breaking Lagrangian. Measuring the Lagrangian requires a lepton collider with a polarized beam, above the threshold for producing some of the superpartners.
\end{abstract}

CP542, Physics Potential and Development of Muon Colliders and Neutrino Factories, edited by David B. Cline (1) 2000 American Institute of Physics 1-56396-970-X/00/\$17.00 


\section{Introduction}

First let us recall the motivations for collider-scale superpartners. The most important is that supersymmetry provides a derivation of the Higgs physics of the Standard Model. The electroweak $\mathrm{SU}(2) \mathrm{x} \mathrm{U}(1)$ symmetry is broken by the Higgs mechanism to accommodate masses for the quarks and leptons and gauge bosons. That breaking cannot be explained within the Standard Model. Supersymmetry with string boundary conditions provides a true explanation. In the Standard Model the Higgs mechanism requires assuming a Higgs field potential energy

$$
V=\mu^{2} h^{2}+\lambda h^{4}
$$

and then assuming that $\mu^{2}$ is negative and $\lambda$ is positive. In supersymmetry the form of the potential is derived, the parameter equivalent to $\mu^{2}$ starts positive at the unification scale, but becomes negative as it runs to the electroweak scale, and $\lambda$ is calculated in terms of gauge couplings.

Thus supersymmetry can explain the origin of the Higgs physics. Further, the explanation works for both fermions and bosons as in the Standard Model. No other approach has been able to do that, and whether an approach can explain the electroweak breaking should be taken as a major test of the validity of any approach to physics beyond the Standard Model.

There are two conditions that must hold for the this explanation to work. One is that the so-called " $\mu$ " term in the superpotential must be absent at tree level, so that it has an induced value of order the supersymmetry breaking masses rather than a value of order the unification scale or the Planck scale. This is immediately satisfied in a string theory since that term enters as a mass term (i.e. of dimension two) and before supersymmetry breaking the low lying spectrum is one of massless strings. Second, the top quark has to have a Yukawa coupling of order one. That again is natural in a string theory where at tree level some Yukawa couplings are of order the gauge couplings. Indeed, the top quark was predicted by this argument to be heavy (compared to the W) in 1982, well before its mass was measured.

There are several additional achievements of supersymmetry that increase the motivation:

- Supersymmetry with light superpartners allows gauge coupling unification.

- Supersymmetry provides a solution of the hierarchy problem, by canceling contributions to the Higgs mass that grow to the largest masses in the theory.

- Supersymmetry provides a candidate for the cold dark matter of the universe, the lightest superpartner (LSP).

- In the Standard Model it is not possible to explain the baryon asymmetry of the universe, while supersymmetry provides at least one way (at the electroweak phase transition) and perhaps others to describe the observed asymmetry. 
- Supersymmetry has predicted three experimental results correctly. One is the top mass mentioned above. The second is the value of $\sin ^{2} \theta_{W}$, using the gauge coupling unification. Both of these predictions were made in the early $1980 \mathrm{~s}$, well before the relevant quantities were measured. The third is the qualitative result of a decade of running at LEP to study Z decays. All supersymmetry effects enter $Z$ decays through loops, unless superpartners are light enough so that direct decays into superpartners can be detected. Therefore, it was predicted that either the superpartners would be seen, or there would be no significant deviations from Standard Model predictions.

If there is no collider scale supersymmetry all of these successes are coincidences. It is also interesting that supersymmetry was not invented to do any of these things. It was invented as a purely theoretical idea, not in response to any puzzle. That it could explain so much physics emerged later as the beautiful idea was studied.

Let us suppose that these successes are not accidental, and we are on the right track. What can we deduce about how to discover superpartners and Higgs bosons if we take this physics seriously? We will assume that nature is indeed described by the supersymmetric Standard Model that produces the above successes. There is no contradiction with not observing superpartners so far - general existing limits are not very constraining.

\section{The Soft-Breaking Lagrangian}

Supersymmetry is a complete quantum field theory, with a known Lagrangian. Although we do not yet understand how supersymmetry is broken, it is possible to write the most general Lagrangian consistent with Lorentz invariance and gauge symmetry. This is called the soft-breaking Lagrangian. The situation is similar to that in the Standard Model before the top quark was observed. We knew how the top quark interacted, just not its mass. For each value of the mass its production cross sections and decays and signatures could be calculated. So we proceed by assuming a mass, doing the calculations, and if experiments are sensitive to that mass then either it is observed or that mass is excluded.

The soft-breaking Lagrangian has a number of parameters. Eventually they have to be measured, just as the quark masses and CKM mixing angles, and lepton masses and mixing angles (which are non-zero if neutrinos have mass), have to be measured. String theory will predict the parameters of the softbreaking Lagrangian once the way to compactify and break supersymmetry is understood, so the measured values will test any theory, and will perhaps point the way to how to compactify and how to break supersymmetry.

The total number of new parameters is 105 for the minimal supersymmetry Standard Model. Sometimes that is said to be a lot of new parameters. But at a similar stage the Standard Model had even more. One can count them various ways, but one way is to assume we know the particles but not the interactions. 
Then a $\mathrm{Z}$ has a vertex with each fermion, and the space-time coupling can be $\mathrm{S}$, V, T, A, P each with complex couplings. For 12 fermions this gives $12 \times 10=120$ parameters. Flavor changing vertices are possible which gives $12 \times 10$ more. The $\mathrm{W}$ couplings to 9 fermion pairs give 90 more. This is 330 parameters not counting WWZ ones. Once there was data that showed the patterns, such as $\mathrm{V}$-A and then gauge invariance, the number quickly dropped to the quark masses, 4 CKM parameters, and two couplings (for the electroweak sector). Similar progress will occur for supersymmetry once there is data showing the patterns of the parameters of the soft-breaking Lagrangian.

\section{What Can We Say About the Superpart- ner Masses?}

The various reasons for expecting supersymmetry to be part of nature, the gauge coupling unification, the hierarchy problem, the explanation of the Higgs mechanism, and LSP cold dark matter, all imply superpartners are light. But none give precise numbers for the superpartner masses. The best we can do is to examine the explanation of the Higgs mechanism. Since it provides an explanation of the $\mathrm{Z}$ and $\mathrm{W}$ masses, it provides an equation of the form

$$
m_{Z}^{2}=\sum C_{i} \tilde{m}_{i}^{2}
$$

The left hand side is the measured value of the Z mass (squared), while the right hand side is a sum of superpartner masses (squared) with calculable coefficients $C_{i}$. The $C_{i}$ can be negative, and some of them are larger than one. If all of the parameters of the soft-breaking Lagrangian are independent, there would be a fine-tuning [1] or unnaturalness if the parameters were not about the same as $m_{Z}$, in which case the superpartners must be very light and accessible at Fermilab or even at LEP.

There are two ways this argument could mislead us. First, it could be that the physical argument that fine tuning just doesn't occur in our mathematical description of nature is somehow wrong. We don't accept that, but it does take an act of faith to believe that we can be guided by requiring the absence of fine tuning. Second, and more important, when we have a real theory, it will predict all of the parameters of the soft-breaking Lagrangian. They will be related to one another. Then there could be cancellations in how the superpartner mass terms combine to give the $\mathrm{Z}$ mass.

We can examine the latter issue in models. We take a D-brane based model, constructed in reference [2], based on the approach of reference [3]. As a stringbased model it has a good motivation, and is less artificial than many ad hoc models, so the conclusions we find should be meaningful. In this model, the 105 new parameters of the soft-breaking Lagrangian are all expressed in terms of 6 physical parameters. We indeed do find that there is less fine tuning for this model than if the parameters were independent. But it remains true [1] that 
$m_{Z}$ is a rapidly increasing function of the remaining mass parameters, and that the masses of the superpartner mass eigenstates are also increasing functions of those masses, so finally it is still true that the fine tuning constraint does imply upper limits on the superpartner masses. Those limits are not large.

The limits depend on $\tan \beta$, and increase with $\tan \beta$. Even for larger $\tan \beta$ they give superpartner and light Higgs masses that are accessible. Gluinos are tightly constrained by these arguments, and should be less than about $500 \mathrm{GeV}$, a mass region that can be explored at Fermilab after a few years of running at expected luminosities. Charginos and neutralinos should be less than about $200 \mathrm{GeV}$, and can be found at Fermilab. The light Higgs boson should be less than about $110 \mathrm{GeV}$, and can be found at Fermilab after about 3 years of running at expected luminosities, or perhaps even at LEP. The conclusion is that IF nature is supersymmetric, and IF supersymmetry indeed provides the explanation for the breaking of the electroweak symmetry, then superpartners and Higgs bosons will be produced and probably detected at Fermilab, or at LEP.

\section{From Superpartners to String Theory}

Experimenters measure the masses of the superpartner mass eigenstates, and their production cross sections times branching ratios, at a scale of about 100 $\mathrm{GeV}$. String theorists work at the Planck scale in 11 dimensions with unbroken supersymmetry. If string theorists write a complete, correct theory of the world with unbroken supersymmetry in 11 dimensions at the Planck scale, its predictions for superpartner masses and interactions will not be deducible. If string theorists propose ways to compactify so the theory is relevant to our $4 \mathrm{D}$ world, and to break the supersymmetry, what they will end up with as the result is the soft-breaking Lagrangian at the relevant scale, probably about the scale where the forces appear to unify, above $10^{16} \mathrm{GeV}$. This Lagrangian is expressed in terms of the soft-breaking parameters, at most one of which actually can be directly measured in an experiment (the gluino mass). Charginos and neutralinos, for example, are the mass eigenstates that emerge from mass matrices formed by the mixing of the superpartners of the gauge bosons and Higgs bosons. To go from the data on charginos and neutralinos to the Lagrangian parameters it is necessary to have measured enough of the mass eigenstates and production rates and branching ratios to invert the equations[4]. Of course this is made more difficult because the data has errors attached. The parameters of the Lagrangian can in general be complex, and if their phases are ignored one can get the wrong answer. Until recently it was thought that the phases had to be small, but in much the same way that in a real theory the fine tuning can change because of relations among the parameters, so the arguments that the phases are small only hold when the parameters are taken to be independent, not when they are related by a theory. The phases may be small, but to know that they will have to be measured. 
An example can make these points clear. The chargino mass matrix is

$$
M_{\tilde{C}}=\left(\begin{array}{cc}
M_{2} e^{i \phi_{2}} & \sqrt{2} M_{W} \sin \beta \\
\sqrt{2} M_{W} \cos \beta & \mu e^{i \phi_{\mu}}
\end{array}\right)
$$

The mass eigenstates $M_{\tilde{C}_{1}}, M_{\tilde{C}_{2}}$ can be obtained from the trace and determinant,

$$
\operatorname{Tr} M_{\tilde{C}}^{\dagger} M_{\tilde{C}}=M_{2}^{2}+\mu^{2}+2 M_{W}^{2}=M_{\tilde{C}_{1}}^{2}+M_{\tilde{C}_{2}}^{2}
$$

$\operatorname{Det} M_{\bar{C}}^{\dagger} M_{\tilde{C}}=M_{2}^{2} \mu^{2}+2 M_{W}^{4} \sin ^{2} 2 \beta-2 M_{W}^{2} M_{2} \mu \sin 2 \beta \cos \left(\phi_{2}+\phi_{\mu}\right)=M_{\tilde{C}_{1}}^{2} M_{\tilde{C}_{2}}^{2}$.

There are similar expressions for the production cross sections and decay branching ratios. These quantities also depend on the soft-breaking parameters here, and typically on others if squarks and sleptons appear in the decay products. Here $M_{2}$ is the soft mass of the SU(2) gaugino, $\mu$ the soft higgsino mass (which is absent in the superpotential because of the string boundary conditions), and $\phi_{2}$ and $\phi_{\mu}$ their phases. For neutralinos the soft mass $M_{1}$ enters, with its phase. In general one cannot assume these phases are equal or small.

Experimenters measure the masses $M_{\tilde{C}_{1}}$ and $M_{\tilde{C}_{2}}$. Obviously one cannot invert the equations to solve for $\tan \beta$ or the phases or soft masses unless sufficiently many observables are measured.

That is the crucial point. Superpartners should be discovered at Fermilab, so one can count the number of observables there and figure out if it is sufficient to measure $\tan \beta$. It is of course necessary to include the decay branching ratios, and therefore the parameters associated with the resulting squarks and sleptons that mediate those decays. Given the limited statistics and backgrounds at Fermilab, and even at LHC, it will not be possible to invert the equations without making assumptions. At a high luminosity lepton collider with polarized beams (NPLC, for Next Polarized Lepton Collider) that is above the threshold for producing some of the superpartners, e.g. the lighter chargino, the two lighter neutralinos, the stop, the lighter Higgs boson, and either producing the pseudoscalar Higgs or putting a good lower limit on its mass, there are enough additional equations from the polarization measurements to invert the equations. Thus the essential measurements needed to go beyond the level of discovery of supersymmetry, and to produce the data needed to test or even to formulate ideas about compactification and supersymmetry breaking, will require the construction of NPLC. The arguments based on electroweak symmetry breaking and fine tuning suggest a lepton collider with total energy of about $600 \mathrm{GeV}$ should be enough to do this.

Before these arguments are completely firm they must be studied by experimenters who do simulations to actually count the observables under realistic conditions, using a plausible general model. With infinite statistics and no backgrounds one can analyze final state polarizations for some decays, but it is unlikely that can be done even at LHC in the real world. My best guess is that it is not possible to solve for $\tan \beta$ at a hadron collider without assumptions 
that may not be true, while it is possible at NPLC. Thus even if superpartners are found at Fermilab, and LHC is a superpartner and Higgs factory, NPLC will be needed to provide the data that will lead to the formulation and testing of string theory for the real world.

\section{ACKNOWLEDGMENTS}

I appreciate the hospitality and support from the organizers, D. Cline in particular, and Joan George and Kevin Lee. I am grateful to J. Wells, S. Mrenna, L. Wang, M. Brhlik, L. Everett, and S. King for discussions and collaborations.

\section{References}

[1] See M. Bastero-Gil, G.L. Kane, and S.King, hep-ph/9910506, to appear in Phys. Lett. for a recent study and references to earlier work.

[2] M. Brhlik et al, Phys. Rev. Letter 83(1999)2124; hep-ph/9908326

[3] L. Ibanez, C. Munoz, and S. Rigolin, hep-ph/9812397

[4] M. Brhlik and G.L. Kane, Phys. Lett. B437(1998)331 